\title{
Numerical simulation of the coagulation dynamics of blood
}

\author{
T. Bodnár ${ }^{\mathrm{a}}$ and A. Sequeira ${ }^{\mathrm{b}} *$ \\ ${ }^{a}$ Department of Technical Mathematics, Faculty of Mechanical Engineering, Czech Technical \\ University, Karlovo Náměstí 13, 12135 Prague 2, Czech Republic; ${ }^{b}$ Department of Mathematics - \\ CEMAT, Instituto Superior Técnico, Av. Rovisco Pais, 1049-001 Lisbon, Portugal
}

(Received 1 May 2007; final version received 4 December 2007)

\begin{abstract}
The process of platelet activation and blood coagulation is quite complex and not yet completely understood. Recently, a phenomenological meaningful model of blood coagulation and clot formation in flowing blood that extends existing models to integrate biochemical, physiological and rheological factors, has been developed. The aim of this paper is to present results from a computational study of a simplified version of this coupled fluid-biochemistry model. A generalized Newtonian model with shearthinning viscosity has been adopted to describe the flow of blood. To simulate the biochemical changes and transport of various enzymes, proteins and platelets involved in the coagulation process, a set of coupled advection-diffusion-reaction equations is used. Three-dimensional numerical simulations are carried out for the whole model in a straight vessel with circular cross-section, using a finite volume semi-discretization in space, on structured grids, and a multistage scheme for time integration. Clot formation and growth are investigated in the vicinity of an injured region of the vessel wall. These are preliminary results aimed at showing the validation of the model and of the numerical code.
\end{abstract}

Keywords: blood coagulation; clot; fibrin; finite-volume; Runge-Kutta

AMS Subject Classification: 35M20; 65L06; 76M12; 92C35; 76Z05; 92C40; 92C50

\section{Introduction}

Haemostasis is a complex physiological process involving an interaction between blood vessels, platelets, coagulation factors, coagulation inhibitors and fibrinolytic proteins. When blood coagulates in a blood vessel during life, the process is called thrombosis. Haemostasis keeps blood flowing while allowing solid clot formation, or thrombosis, to prevent blood loss from sites of vascular damage. The haemostatic system preserves intravascular integrity by achieving a balance between haemorrhage and thrombosis.

Blood platelets participate in both haemostasis and thrombosis. The first stage of thrombogenesis is platelets activation, followed by platelets aggregation, adhesion and blood coagulation, with the formation of clots. Blood platelets can be activated by prolonged exposure to high or rapid increase in shear stress that lead to erythrocytes and platelets damage $[10,15]$. This is due to mechanical vascular injuries or endothelial disturbance, alterations in the blood composition, fissuring of atherosclerotic plaques as well as to the contact of blood with the surfaces of medical devices. Numerous experimental studies recognize that clot formation rarely occurs in regions of parallel flow,

*Corresponding author. Email: adelia.sequeira@math.ist.utl.pt 
but primarily in regions of stagnation point flows, within blood vessel bifurcations, branching and curvatures.

Following endothelial disruption, there is an immediate reaction that promotes vasoconstriction, minimizing vessel diameter and diminishing blood loss. Vasoconstriction slows blood flow, enhancing platelet adhesion and activation. During activation, platelets undergo intrinsic and extrinsic mechanisms leading to a series of chemical and morphological changes. Organelles contained in the platelet cytoplasm bind to collagen (exposed by arterial damage), release their contents of cytoplasmic granules containing serotonin, adenosine diphosphate (ADP) and platelet-activating factors and platelets become spheroids in shape. Additional platelets attracted by ADP are activated, interact with plasma proteins like fibrinogen and fibrin and promote platelet aggregation and adhesion to sub-endothelial tissue. This results in the formation of haemostatic plugs and concludes the primary step in haemostasis. However, when the concentration of activators exceeds a threshold value, platelet aggregates that are formed by this process can break up, damaging the platelets and causing aggregation at locations other than at the site of damage.

The final haemostatic mechanism or secondary haemostasis is coagulation (clot formation) which involves a very complex cascade of enzymatic reactions (Figure 1). Thrombin is the bottom enzyme of the coagulation cascade. Prothrombin activator converts prothrombin to thrombin. Thrombin activates platelets that release ADP which

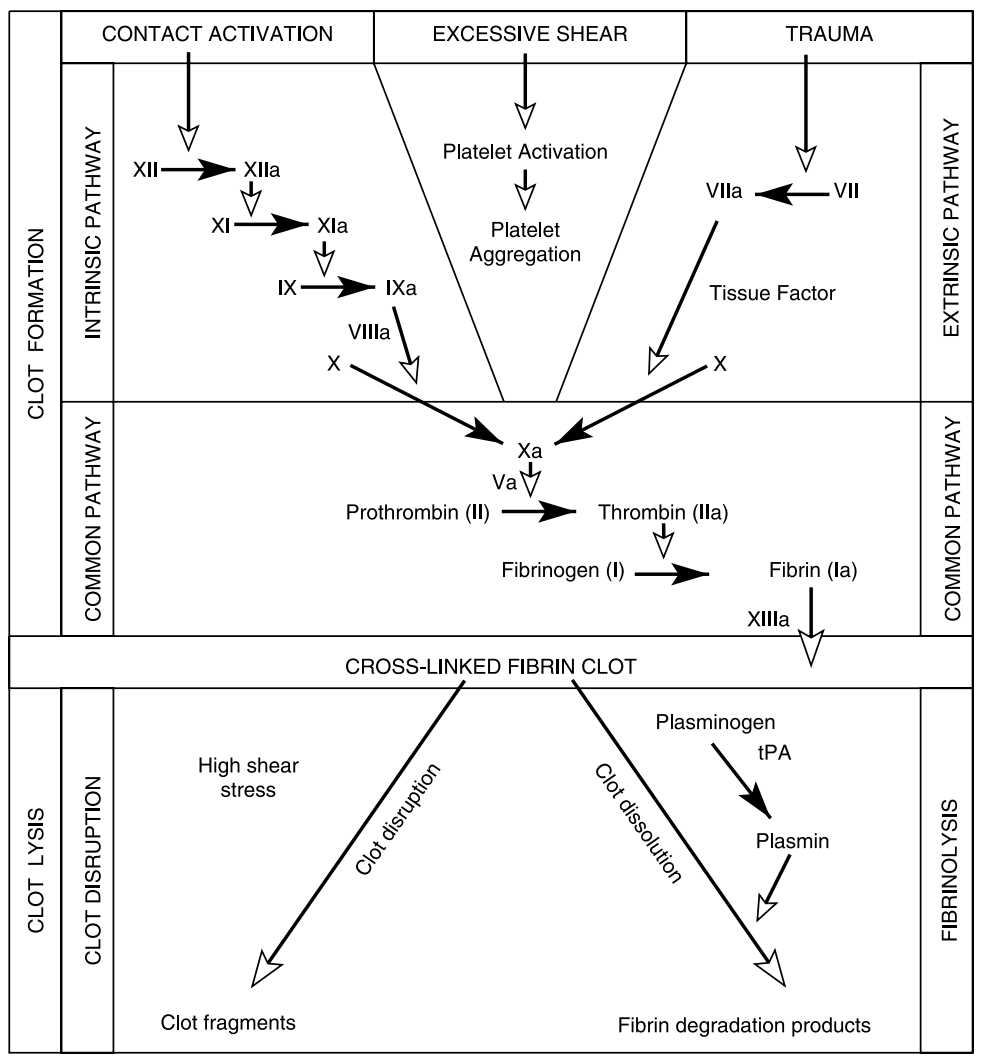

Figure 1. Schematic of the cascade of events during clot formation and lysis. 
lead in turn to the activation of other platelets. The primary role of thrombin is to convert fibrinogen, a blood protein, into polymerized fibrin, stabilizing the adhered platelets and forming a viscoelastic blood clot (or thrombus) (e.g. [21,24]).

The clot attracts and stimulates the growth of fibroplasts and smooth muscle cells within the vessel wall, and begins the repair process which ultimately results in fibrinolysis and in the dissolution of the clot (clot lysis). Clot dissolution can also occur due to mechanical factors such as high shear stress. In practice, a blood clot can be continuously formed and dissolved. Generally, many factors affect its structure, including the concentration of fibrinogen, thrombin, albumin, platelets and red bloodcells, and other not specified factors which determine cross-linked structure of the fibrin network. At the end of the haemostatic process, normal blood flow conditions are restored. However, some abnormal haemodynamic and biochemical conditions of flowing blood, related to inadequate levels or dysfunction of the haemostatic system, can lead to pathologies like thromboembolic or bleeding disorders which are of great clinical importance. The mechanism of platelet activation and blood coagulation is quite complex. Recent reviews detailing the structure of the blood coagulation system are available for example in Refs. [3,24].

Whole blood is a concentrated suspension of formed cellular elements that includes red blood cells (RBCs) or erythrocytes, white blood cells or leukocytes and platelets or thrombocytes, representing approximately $45 \%$ of the volume in human blood. These cellular elements are suspended in plasma, an aqueous ionic solution of low viscosity. Plasma behaves as a Newtonian fluid but whole blood has non-Newtonian properties. In the large vessels where shear rates are high enough, it is also reasonable to assume that blood has a constant viscosity and a Newtonian behaviour. However, in smaller vessels, or in some disease conditions, the presence of the cells induces low shear rate and whole blood exhibits remarkable non-Newtonian characteristics, like shear-thinning viscosity, thixotropy, viscoelasticity and possibly a yield stress. This is largely due to erythrocytes behaviour, namely their ability to aggregate into microstructures (rouleaux) at low shear rates, their deformability into an infinite variety of shapes without changing volume and their tendency to align with the flow field at high shear rates [8,25].

The most well-known non-Newtonian characteristic of blood is its shear-thinning behaviour [7,9]: at low shear rates, blood seems to have a high apparent viscosity (due to RBCs aggregation) while at high shear rates there is a reduction in the blood's viscosity (due to RBCs deformability). Stress relaxation properties of blood arise from its ability to store and release elastic energy from $\mathrm{RBC}$ networks and are mainly related to the viscoelastic nature of the RBC membrane. The viscoelastic behaviour of blood is less important at higher shear rates [27]. An understanding of the coupling between the blood composition and its physical properties is essential for developing suitable constitutive models to describe blood behaviour (see the recent reviews [22,23]).

While there has been a considerable research effort in blood rheology, the constitutive models have thus far focused on the aggregation and deformability of the RBCs, ignoring the role of platelets in the flow characteristics. However, platelets, which are much smaller than erythrocytes (approximately $6 \mu \mathrm{m}^{3}$ in size as compared to $90 \mu \mathrm{m}^{3}$ ) and form a small fraction of the particulate matter in human blood (around 3\% in volume) are among the most sensitive of all the components of blood to chemical and physical agents and play an important role in coagulation, as already discussed. In the last two decades, mathematical modelling and computer simulation research have emerged as useful tools, supplementing experimental data and analysis and giving new insights in the studies of the regulation of the coagulation cascade in clinical applications and device design. Reliable 
phenomenological models that can predict regions of platelet activation and deposition (either in artificial devices or in arteries) have the potential to help optimize design of such devices and also identify regions of the arterial tree susceptible to the formation of thrombotic plaques and possible rupture in stenosed arteries. Most of the models that are currently in use neglect some of the biochemical or mechanical aspects involved in the complex phenomena of blood coagulation and must be considered as first approaches to address this oversight, see, for example [5,6,12,16,18,29,31,32]. Other relevant biochemistry related references can be found in the recent review paper by Rajagopal and Lawson [20]. Anand et al. [2-4] recently developed a phenomenological comprehensive model for clot formation and lysis in flowing blood that extends existing models to integrate biochemical, physiological and rheological factors. In what follows, we present some preliminary computational results for a simplified version of this model where the viscoelasticity of blood is not considered. Section 2 is devoted to a description of the coupled blood flow-biochemistry mathematical model. The numerical methods and adopted stabilization techniques are briefly outlined in Section 3. Preliminary results of the three-dimensional simulations predicted by the simpler model are presented in Section 4. Finally, some comments on the limitations and extensions of the model are also included.

\section{Mathematical model}

\subsection{Blood flow equations}

An incompressible generalized Newtonian model with shear-thinning viscosity has been adopted to describe the flow of blood. We denote by $\mathbf{u}(x, t)$ and $p(x, t)$ the blood velocity and pressure defined in the domain $\Omega$, the vascular lumen, with $t \geq 0$. The application of the physical principles of momentum and mass conservation leads to the equations defined in $\Omega$

$$
\rho\left(\frac{\partial \mathbf{u}}{\partial t}+\mathbf{u} \cdot \nabla \mathbf{u}\right)=-\nabla p+\operatorname{div} \tau(\mathbf{u}) \quad \operatorname{div} \mathbf{u}=0,
$$

completed with appropriate initial and boundary conditions. Here, $\rho$ is the fluid density and $\tau(\mathbf{u})$ is the deviatoric stress tensor, proportional to the symmetric part of the velocity gradient, given by

$$
\tau(\mathbf{u})=2 \mu(\dot{\gamma}) \mathrm{D},
$$

where $\mathrm{D}=\left(\nabla \mathbf{u}+\nabla \mathbf{u}^{\mathrm{T}}\right) / 2$ is the stretching tensor, $\dot{\gamma}=2 \sqrt{\mathrm{D}: \mathrm{D}}$ is the shear rate (a measure of the rate of shear deformation) and $\mu(\dot{\gamma})$ is the shear dependent viscosity function which decreases with increasing shear rate and can be written in the following general form

$$
\mu(\dot{\gamma})=\mu_{\infty}+\left(\mu_{0}-\mu_{\infty}\right) F(\dot{\gamma})
$$

or, in non-dimensional form as

$$
\frac{\mu(\dot{\gamma})-\mu_{\infty}}{\mu_{0}-\mu_{\infty}}=F(\dot{\gamma}) .
$$

Here, $\mu_{0}=\lim _{j \mapsto 0} \mu(\dot{\gamma})$ and $\mu_{\infty}=\lim _{j \mapsto \infty} \mu(\dot{\gamma})$ are the asymptotic viscosity values at low and high shear rates and $F(\dot{\gamma})$ is a shear dependent function satisfying the following natural limit conditions $\lim _{\dot{\gamma} \rightarrow 0} F(\dot{\gamma})=1$ and $\lim _{\dot{\gamma} \rightarrow \infty} F(\dot{\gamma})=0$. The generalized Cross model has 
frequently been used for blood and has been adopted here. The corresponding viscosity function is written as

$$
\frac{\mu(\dot{\gamma})-\mu_{\infty}}{\mu_{0}-\mu_{\infty}}=\frac{1}{\left(1+(\lambda \dot{\gamma})^{b}\right)^{a}}
$$

Using nonlinear regression analysis, it is possible to fit viscosity functions against blood viscosity experimental data and obtain the corresponding parameters $\lambda, a$ and $b$. However, blood viscosity is quite sensitive to a number of factors including haematocrit, temperature, plasma viscosity, age of RBCs, exercise level, gender or disease state, and care must be taken in selecting blood parameters for blood flow simulations. Here, we have adopted the following values of the material constants (see Ref. [17]):

$$
\mu_{0}=1.6 \cdot 10^{-1} \mathrm{~Pa} \cdot \mathrm{s} \quad \mu_{\infty}=3.6 \cdot 10^{-3} \mathrm{~Pa} \cdot \mathrm{s} \quad a=1.23 \quad b=0.64 \quad \lambda=8.2 \mathrm{~s} .
$$

The viscosity function (4) with these values is represented in Figure 2.

\subsection{Equations governing the cascade of biochemical reactions}

The model includes not only rheological factors but also biochemical indicators that are essential to describe coagulation and fibrinolysis dynamics and consequently the formation, growth and dissolution of clots. It is based on the model introduced in Ref. [3] and further developed and refined in Ref. [2]. A set of twenty-three coupled advectiondiffusion-reaction equations, describing the evolution in time and space of various enzymes and zymogenes, proteins, inhibitors, tissue plasminogen activator and fibrin/fibrinogen (Table 1) involved in the extrinsic pathway of the blood coagulation process and fibrinolysis in quiescent plasma, takes the following form

$$
\frac{\partial \mathrm{C}_{i}}{\partial t}+\operatorname{div}\left(\mathrm{C}_{i} \mathbf{u}\right)=\operatorname{div}\left(\mathrm{D}_{i} \nabla \mathrm{C}_{i}\right)+\mathrm{R}_{i} \quad i=1, \ldots, 23 .
$$

In these equations, $\mathrm{C}_{i}$ stands for the concentration of $i$ th reactant, $\mathrm{D}_{i}$ denotes the corresponding diffusion coefficient (which could be a function of the shear rate) and $\mathbf{u}$ is the velocity field. $\mathrm{R}_{i}$ are the nonlinear reaction terms that represent the production or depletion of $\mathrm{C}_{i}$ due to the enzymatic cascade of reactions. The specific form of the reaction terms, the diffusion coefficients and corresponding parameters for each of the 23-model

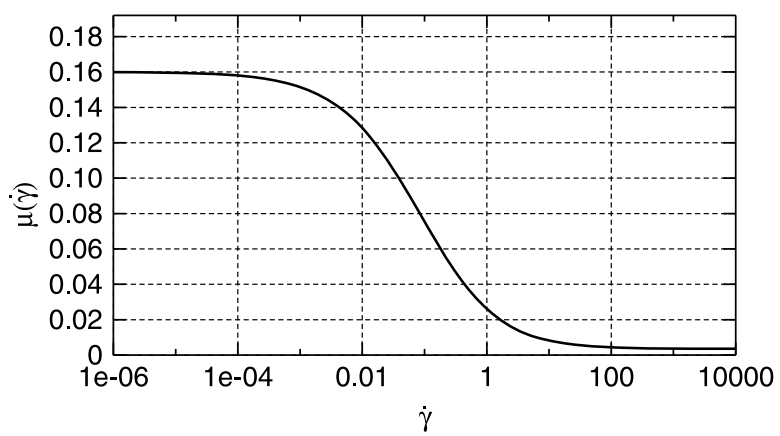

Figure 2. Variation of the apparent viscosity of blood as a function of the shear rate for the generalized Cross model with physiological parameters given by (5). 
Table 1. Constituents involved in the coagulation cascade and fibrinolysis.

\begin{tabular}{ll}
\hline Species & Names \\
\hline$I X a$ & ?Enzym? \\
$I X$ & Zymogen IX \\
$V I I I a$ & Enzym VIIIa \\
$V I I I$ & Zymogen VIII \\
$V a$ & Enzym Va \\
$V$ & Zymogen V \\
$X a$ & Enzym Xa \\
$X$ & Zymogen X \\
$I I a$ & Thrombin \\
$I I$ & Prothrombin \\
$I a$ & Fibrin \\
$I$ & Fibrinogen \\
$X I a$ & Enzym XIa \\
$X I$ & Zymogen XI \\
$A T I I I$ & AntiThrombin-III \\
$T F P I$ & Tissue factor pathway inhibitor \\
$A P C$ & Active protein C \\
$P C$ & Protein C \\
$L 1 A T$ & $\alpha_{1}$-AntiTrypsin \\
$t P A$ & Tissue plasminogen activator \\
$P L A$ & Plasmin \\
$P L S$ & Plasminogen \\
$L 2 A P$ & $\alpha_{2}$-AntiPlasmin \\
$Z$ & Tenase \\
$W$ & Prothrombinase \\
\hline &
\end{tabular}

Equations (6) is shown in Tables 2 and 3. In order to keep the model readable, symbol [.] is used to denote the concentration of a reactant and to distinguish it from its name (e.g. [ATIII] denotes the concentration of AntiThrombin III (ATIII)).

Equations (6) are complemented with appropriate initial and flux boundary conditions involving the concentration of various species at an injured site of the vascular wall (see Section 4.1.2). A schematic representation of these reactions, including boundary conditions is given in Figure 3. This figure also includes interactions with two other chemical species $Z$ (tenase) and $W$ (prothrombinase), see Table 6. Separate convectiondiffusion reactions are not written for the corresponding concentrations, since they are embedded in the equations for VIIIa, IXa,Va and $\mathrm{Xa}$. Their values can be obtained through the relations $[\mathrm{Z}]=[\mathrm{VIIIa}][I \mathrm{IX}] / K_{d Z},[\mathrm{~W}]=[\mathrm{Va}][\mathrm{Xa}] / K_{d W}[2]$, respectively.

\subsection{Clot modeling}

Under normal conditions, no clot exists and we assume that the presence of all these constituents does not affect the velocity of the bulk flow. Clot formation occurs when an activation threshold in the flux boundary conditions, related to the concentration of tissue factor (TF-VIIa complex released from sub-endothelium) is exceeded and the clotting cascade is initiated, which results in the increase of fibrin concentration $[I a]$ in the clotting area (see Section 4.1.2). Clot growth is determined by tracking in time the extent of the flow region where fibrin concentration $C_{F}$ equals or exceeds a specific critical value $C_{\text {Clot }}$. The clot region can thus be defined as a region where the fibrin concentration is higher than in the normal blood. The main features of the modelling approach are schematically shown in Figure 4. 
Table 2. Reaction terms and diffusion coefficients.

\begin{tabular}{llc}
\hline & \multicolumn{1}{c}{ Reaction terms $\left(\mathrm{R}_{i}\right)$} & \\
Species & \multicolumn{1}{c}{ Diffusion coefficients } \\
$\mathrm{D}_{i}\left(\mathrm{~cm}^{2} / \mathrm{s}\right)$
\end{tabular}

An important assumption of the coupling strategy for blood and clot is to assume that the corresponding constitutive models are similar, but their viscosities have different values. This can be done introducing a viscosity factor related to the fibrin concentration around the injured vessel wall region. More precisely, when fibrin concentration is close to zero, the local viscosity is equal to the viscosity of blood (thus the viscosity factor is equal to 1 ). As the fibrin concentration grows, the viscosity increases linearly up to a certain threshold $\mathrm{C}_{\mathrm{Clot}}$ and the viscosity factor is equal to $\mu^{*}$ (Figure 5). The parameters $\mu^{*}$ and $\mathrm{C}_{\mathrm{Clot}}$ should be chosen to mimic the clot properties. In our study, we have used $\mu^{*}=100$ and $\mathrm{C}_{\mathrm{Clot}}=1000 \mathrm{nM}$ corresponding to a viscosity of the clot region 100 times higher than that of the blood.

This model function of the viscosity factor based on fibrin concentration as well as the values used to simulate the clot growth look reasonable, but can easily been improved if experimental data are available.

\section{Numerical methods}

The numerical solution of the coupled fluid-biochemistry model given by Equations (1)(2)-(6) is obtained using a three-dimensional code based on a finite-volume semidiscretization in space, on structured grids, where both inviscid and viscous flux integrals are evaluated using centred cell numerical fluxes. An explicit multistage Runge-Kutta scheme is used for time integration. The same method is applied for both the flow and the biochemical solutions of the coupled model. The coupling is carried out by means of an iterative process at each time step. The flow field is first computed using Equations (1)-(2) 
Table 3. Reaction kinetics parameters.

\begin{tabular}{|c|c|c|}
\hline Parameters & Values & Units \\
\hline$k_{9}$ & 11.0 & $\min ^{-1}$ \\
\hline$K_{9 M}$ & 160 & $\mathrm{nM}$ \\
\hline$h_{9}^{A 3^{A U}}$ & 0.0162 & $\mathrm{nM}^{-1} \min ^{-1}$ \\
\hline$k_{8}$ & 194.4 & $\min ^{-1}$ \\
\hline$K_{8 M}$ & 112,000 & $\mathrm{nM}$ \\
\hline$h_{8}$ & 0.222 & $\min ^{-1}$ \\
\hline$h_{C 8}$ & 10.2 & $\min ^{-1}$ \\
\hline$H_{C 8 M}$ & 14.6 & $\mathrm{nM}$ \\
\hline$k_{5}$ & 27.0 & $\min ^{-1}$ \\
\hline$K_{5 M}$ & 140.5 & $\mathrm{nM}$ \\
\hline$h_{5}$ & 0.17 & $\min ^{-1}$ \\
\hline$h_{C 5}$ & 10.5 & $\min ^{-1}$ \\
\hline$H_{C 5 M}$ & 14.6 & $\mathrm{nM}$ \\
\hline$k_{10}$ & 2391 & $\min ^{-1}$ \\
\hline$K_{10 M}$ & 160.0 & $\mathrm{nM}$ \\
\hline$h_{10}$ & 0.347 & $\mathrm{nM}^{-1} \min ^{-1}$ \\
\hline$h_{T F P I}$ & 0.48 & $\mathrm{nM}^{-1} \min ^{-1}$ \\
\hline$k_{2}$ & 1344.0 & $\min ^{-1}$ \\
\hline$K_{2 M}$ & 1060.0 & $\mathrm{nM}$ \\
\hline$h_{2}$ & 0.714 & $\mathrm{nM}^{-1} \min ^{-1}$ \\
\hline$k_{1}$ & 3540 & $\min ^{-1}$ \\
\hline$K_{1 M}$ & 3160 & $\mathrm{nM}$ \\
\hline$h_{1}$ & 1500 & $\min ^{-1}$ \\
\hline$H_{1 M}$ & 250,000 & $\mathrm{nM}$ \\
\hline$k_{11}$ & 0.0078 & $\min ^{-1}$ \\
\hline$K_{11 M}$ & 50.0 & $\mathrm{nM}$ \\
\hline$h_{11}^{A 3}$ & $1.6 \times 10^{-3}$ & $\mathrm{nM}^{-1} \min ^{-1}$ \\
\hline$h_{11}^{L 1}$ & $1.3 \times 10^{-5}$ & $\mathrm{nM}^{-1} \min ^{-1}$ \\
\hline$k_{P C}$ & 39.0 & $\min ^{-1}$ \\
\hline$K_{P C M}$ & 3190.0 & $\mathrm{nM}$ \\
\hline$h_{P C}$ & $6.6 \times 10^{-7}$ & $\mathrm{nM}^{-1} \min ^{-1}$ \\
\hline$k_{P L A}$ & 12.0 & $\min ^{-1}$ \\
\hline$K_{P L A M}$ & 18.0 & $\mathrm{nM}$ \\
\hline$h_{P L A}$ & 0.096 & $\mathrm{nM}^{-1} \min ^{-1}$ \\
\hline$k_{A P}^{A P}$ & 18.0 & $\mathrm{nM}^{-1} \min ^{-1}$ \\
\hline$h_{A P}^{H I a}$ & 30.0 & $\min ^{-1}$ \\
\hline$K_{d Z}$ & 0.56 & $\mathrm{nM}$ \\
\hline$K_{d W}$ & 0.1 & $\mathrm{nM}$ \\
\hline
\end{tabular}

with a given viscosity, and next inserted into (6) to obtain the concentrations (including that of fibrin). The local viscosity is updated by a factor which depends on the calculated fibrin concentration in the clotting area and the flow field is recomputed in the next time step using the updated viscosity.

An artificial compressibility formulation (see, e.g. [28]) is used to calculate the pressure and to enforce the divergence-free constraint. Continuity Equation $(1)_{2}$ is modified by adding the time-derivative of pressure properly scaled by the artificial speed of sound $\mathfrak{c}$, as follows:

$$
\frac{1}{c^{2}} \frac{\partial p}{\partial t}+\operatorname{div} \mathbf{u}=0
$$




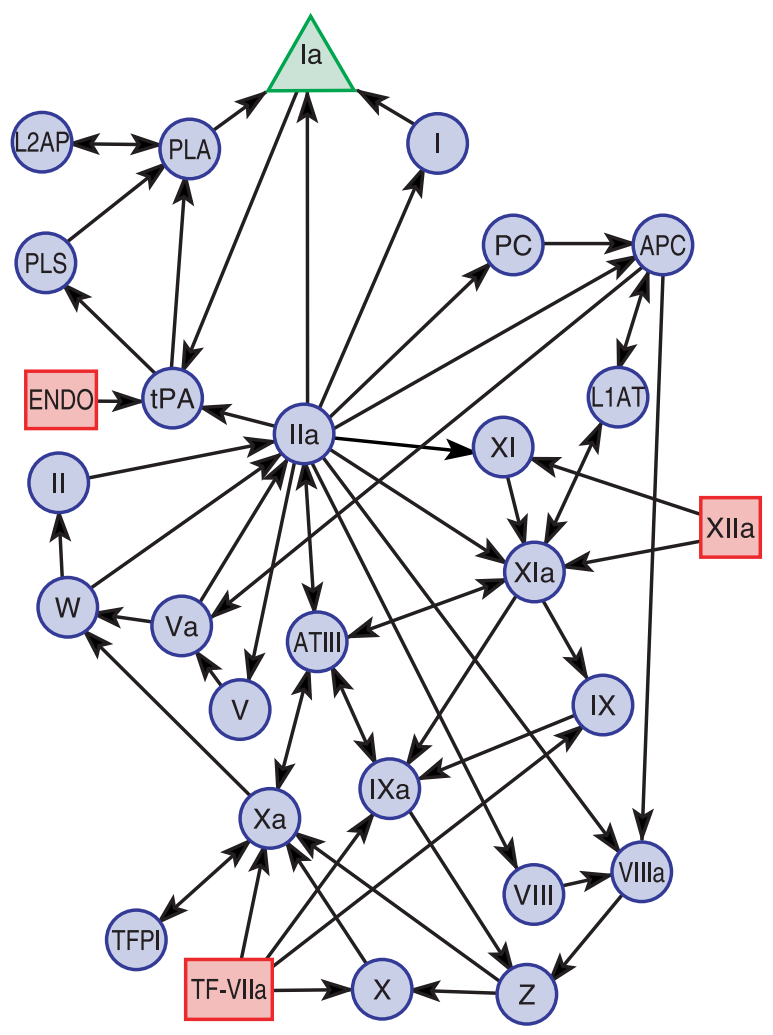

Figure 3. Schematic of the selected reactions involved in the extrinsic pathway of the blood coagulation process.

This non-physical modification of the continuity equation is often used in steady flow simulations. In the present case, the sub-iterative procedure based on the artificial compressibility method is only used to enforce the divergence-free constraint before simulating the biochemical concentrations. This could be justified by the quasi-steady nature of the flow, due to the slow evolution in time of the local viscosity. Thus, it looks acceptable to assume the flow to be described by a steady generalized Newtonian system at each time step.

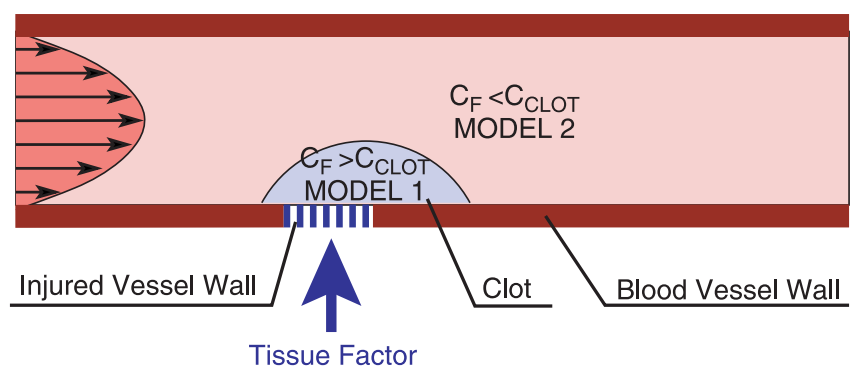

Figure 4. Clot modeling. 


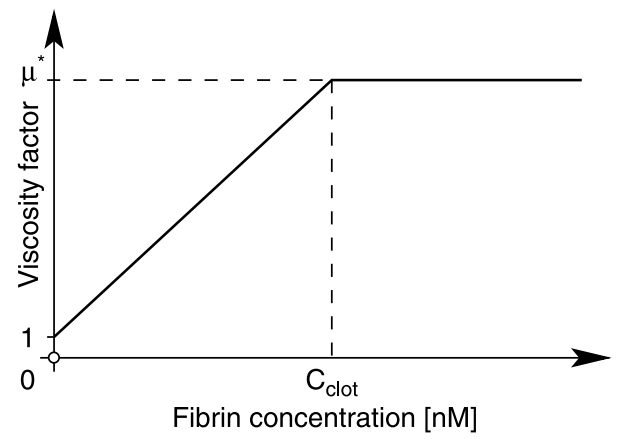

Figure 5. Clot viscosity behaviour.

\subsection{Space discretization}

The computational mesh is structured and consists of hexahedral primary control volumes. To evaluate the viscous fluxes also, dual finite volumes are needed. They have octahedral shape and are centred around the corresponding primary cell faces. Figure 6 shows a schematic representation of this configuration. The same grid is used for the calculation of both flow field and biochemical concentrations. Thus, it is natural to use the same method for solving both problems simultaneously. The algorithm described below for the flow variables $\mathbf{W}$, can thus be directly applied to the calculation of the concentrations $\mathrm{C}_{i}$.

The system of generalized NS Equations (1)-(2) can be rewritten in the vector form. Here, we use $\mathrm{W}$ to denote the vector of unknowns (including pressure). Vectors $\mathrm{F}, \mathrm{G}$ and $\mathrm{H}$ denote the inviscid fluxes in $x, y, z$ directions, while $\mathrm{R}, \mathrm{S}$ and $\mathrm{T}$ stand for their viscous counterparts. Using this notation, the spatial finite-volume semi-discretization in the computational cell $D \equiv D_{i, j, k}$ can be written in the following form:

$$
\frac{\partial \mathrm{W}}{\partial t}=\frac{-1}{|D|} \oint_{\partial D}[(\mathrm{~F}-\mathrm{R}),(\mathrm{G}-\mathrm{S}),(\mathrm{H}-\mathrm{T})] \cdot \hat{\boldsymbol{v}} \mathrm{d} S .
$$

Here, $\hat{\boldsymbol{v}}$ denotes the outer unit normal vector of the cell boundary and $\mathrm{d} S$ is the surface element of this boundary. Equation (8) can be rewritten in operator form:

$$
\frac{\partial \mathbf{W}_{i j k}}{\partial t}=-\mathcal{L} \mathbf{W}_{i, j, k}
$$

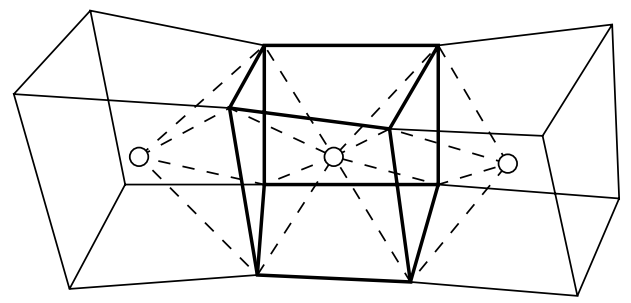

Figure 6. Finite-volume grid in 3D. 
where $\mathcal{L}$ stands for the finite-volume discretization operator. This operator is still exact at this stage and must be properly discretized.

The inviscid flux integral can be approximated using centred cell fluxes, e.g. the value of the flux $F$ on the cell face with index $\ell=1$ is computed as the average of cell-centred values from both sides of this face:

$$
\mathrm{F}_{1}^{n}=\frac{1}{2}\left[\mathrm{~F}\left(\mathbf{W}_{i, j, k}^{n}\right)+\mathrm{F}\left(\mathbf{W}_{i+1, j, k}^{n}\right)\right] .
$$

The contribution of inviscid fluxes is finally summed up over the cell faces $\ell=1, \ldots, 6$. In this way, we can write the inviscid flux approximation:

$$
\oint_{\partial D} \mathrm{~F} \nu^{x} \mathrm{~d} y \mathrm{~d} z \approx \sum_{\ell=1}^{6} \mathrm{~F}_{\ell} \nu_{\ell}^{x} S_{\ell}
$$

The discretization of viscous fluxes is more complicated since vectors $R, S, T$ involve the derivatives of the velocity components that need to be approximated at cell faces. This can be done using a dual finite-volume grid, with octahedral cells, centred around the corresponding faces (Figure 7). The evaluation of the velocity gradient components is then replaced by the evaluation of the surface integral over the dual volume boundary. Finally, this surface integral is approximated by a discrete sum over the dual cell faces (with indices $m=1, \ldots, 8)$. For example, if we want to evaluate the first component of the viscous flux $\mathrm{R}_{1}$ (i.e. to approximate $u_{x}$ ) at the cell face $l=1$, we must proceed in the following way:

$$
u_{x} \approx \oint_{\partial \tilde{D}} u \nu^{x} \mathrm{~d} y \mathrm{~d} z \approx \sum_{m=1}^{8} u_{m} \nu_{m}^{x} S_{m}
$$

The outer normal to the dual cell faces must be properly approximated $\nu^{x} \approx \nu_{m}^{x}$. The values of the velocity components in the middle nodes of these faces are taken as an average of the values in the corresponding vertices.

\subsection{Time integration}

After space discretization, the problem is now in the semi-discrete form:

$$
\frac{\mathrm{dW}_{i j k}}{\mathrm{~d} t}=-\mathcal{L} \mathrm{W}_{i, j, k}
$$

where $\mathcal{L}$ is the approximate space discretization operator.
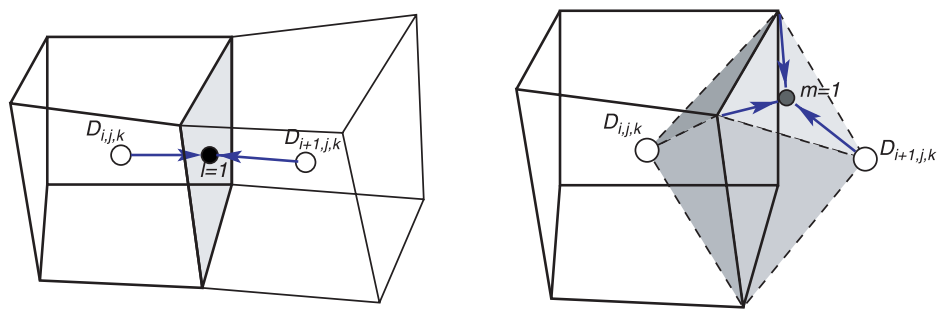

Figure 7. Inviscid flux (left) and viscous flux (right) discretizations. 
This system of ordinary differential equations for $\mathrm{W}$ (or concentration $\mathrm{C}_{i}$ ) can be solved, e.g. by the Runge-Kutta multistage method. For simplicity, we use the same time integration method for both, flow field and concentrations. One of the simplest RungeKutta methods can be written in the form:

$$
\begin{aligned}
& \mathbf{W}_{i, j, k}^{(0)}=\mathbf{W}_{i, j, k}^{n} \\
& \mathbf{W}_{i, j, k}^{(r+1)}=\mathbf{W}_{i, j, k}^{(0)}-\alpha_{(r)} \Delta t \mathcal{L} \mathbf{W}_{i, j, k}^{(r)} \\
& \mathbf{W}_{i, j, k}^{n+1}=\mathbf{W}_{i, j, k}^{(m)} .
\end{aligned}
$$

Here, $r=1, \ldots, m$ is related to the $m$-stage method. The three-stage explicit RK scheme has coefficients: $\alpha_{(1)}=1 / 2, \alpha_{(2)}=1 / 2$ and $\alpha_{(3)}=1$. This method is useful and efficient with minimum requirements on the storage space associated with intermediate stages.

The efficiency and robustness of the method could be further increased modifying the above algoritm. The modification used in the numerical simulations presented in this paper follows the Runge-Kutta time integration procedures outlined in Ref. [14] and further refined in Ref. [13]. The idea behind this modified approach lies in splitting the space discretization operator into its inviscid and viscous parts. The inviscid operator is evaluated at each Runge-Kutta stage, while the viscous operator is evaluated in just a few more stages. This corresponds to the use of different Runge-Kutta coefficients for time integration of inviscid and viscous fluxes. The modified algorithm can thus be written in the form:

$$
\begin{aligned}
& \mathbf{W}_{i, j, k}^{(0)}=\mathbf{W}_{i, j, k}^{n} \\
& \mathbf{W}_{i, j, k}^{(r+1)}=\mathbf{W}_{i, j, k}^{(0)}-\alpha_{(r)} \Delta t\left(\mathcal{Q}^{(r)}+\mathcal{D}^{(r)}\right) \\
& \mathbf{W}_{i, j, k}^{n+1}=\mathbf{W}_{i, j, k}^{(m)} .
\end{aligned}
$$

Here, the space discretization operator at stage $(r)$ is split as:

$$
\mathcal{L} \mathbf{W}_{i, j, k}^{(r)}=\mathcal{Q}^{(r)}+\mathcal{D}^{(r)}
$$

The inviscid flux $\mathcal{Q}$ is evaluated in the usual way at each stage

$$
\mathcal{Q}^{(r)}=\mathcal{Q} \mathbf{W}_{i, j, k}^{(r)} \quad \text { with } \quad \mathcal{Q}^{(0)}=\mathcal{Q} \mathbf{W}_{i, j, k}^{n} .
$$

The viscous flux $\mathcal{D}$ uses a blended value from the previous and the actual stages according to the following rule:

$$
\mathcal{D}^{(r)}=\beta_{(r)} \mathcal{D} \mathbf{W}_{i, j, k}^{(r)}+\left(1-\beta_{(r)}\right) \mathcal{D}^{(r-1)} \quad \text { with } \quad \mathcal{D}^{(0)}=\mathcal{D} \mathbf{W}_{i, j, k}^{n}
$$


The coefficients $\alpha_{(r)}$ and $\beta_{(r)}$ are chosen to guarantee a large enough stability region for the Runge-Kutta method. The following set of coefficients was used in this study:

$$
\begin{array}{ll}
\alpha_{(1)}=1 / 3 & \beta_{(1)}=1 \\
\alpha_{(2)}=4 / 15 & \beta_{(2)}=1 / 2 \\
\alpha_{(3)}=5 / 9 & \beta_{(3)}=0 \\
\alpha_{(4)}=1 & \beta_{(4)}=0
\end{array} .
$$

We realize that only two evaluations of the dissipative terms are needed when this four-stage method is used. This characteristic of the method significantly saves the amount of calculations while retaining the advantage of a large stability region. Further admissible sets of coefficients together with comments on the increase of the efficiency and robustness of the corresponding methods can be found in Refs. [13,14] and the references therein.

\subsection{Numerical stabilization}

A well-known property of central schemes is that, in the presence of strong gradients, nonphysical oscillations appear in the solution. Procedures aiming at stabilizing the discrete solution and to avoid spurious pressure modes can be accomplished according to several criteria.

In the present simulations, we use a pressure stabilization method that consists in adding a pressure dissipation term (Laplacian) on the right-hand side of the modified continuity Equation (7). This term takes the form:

$$
Q_{i, j, k}=\frac{1}{\left|D_{i, j, k}\right|} \sum_{\ell=1}^{2 N} \frac{p_{\ell}-p_{i, j, k}}{b_{\ell}} S_{\ell} .
$$

Here, $\ell$ denotes the control volume cell face index, $p_{\ell}$ is the pressure in the corresponding neighbouring cell and $S_{\ell}$ is the cell face area. The value $b_{\ell}$ has the dimension of velocity and represents the maximal convective velocity in the domain and local diffusive velocity.

$$
b_{\ell}=\max \left(\sqrt{u_{1}^{2}+u_{2}^{2}+u_{3}^{2}}\right)+\frac{2 \nu}{L_{\ell}} .
$$

Symbol $L_{\ell}$ corresponds to a distance between the current and the neighbouring cell centres.

It is possible to show that on the uniform cartesian mesh with cells of size $\delta x$, this term is given by:

$$
Q=\frac{\delta x}{2 b} \Delta p .
$$

This stabilization method has some advantages over the classical artificial diffusion applied to the velocity components. The artificial effects induced by the pressure dissipation term do not interfere with the physical shear dependent viscosity. Moreover, this stabilization term contains only second derivatives of the pressure and will vanish if pressure is a linear function of space coordinates. See also Ref. [19] for other stabilization approaches.

Finally, it is important to observe that since the diffusion coefficients in the advectiondiffusion-reaction equations of the biochemistry model are extremely small, the use of a central space-discretization, with no internal numerical diffusion, seems to be a natural 
choice. To prevent the appearance of numerical oscillations typically associated to central schemes, a special non-linear filter was also used at each iteration to postprocess the concentration fields. This filtering technique described in Ref. [11] was further studied in Ref. [26].

\section{Numerical simulations and preliminary results}

The computational domain consists of a segment of a rigid-walled cylindrical vessel with diameter $D=2 R=6.2 \mathrm{~mm}$ and length $L=10 R=31 \mathrm{~mm}$. The whole domain is discretized using a structured multiblock mesh with hexahedral cells as shown in Figure 8. The grid is fitted to the vessel wall in order to capture the near-wall gradients of the velocity and concentration fields. The grid has $10 \times 10 \times 60$ cells in the core block, while there are $40 \times 20 \times 60$ cells in the outer, near-wall block.

\subsection{Boundary and initial conditions}

\subsubsection{Velocity and pressure}

A fully developed velocity profile, with mean velocity $U=3.1 \mathrm{~cm} / s$ (related to the vessel length by $U=L / s=10 R / s$ ), is prescribed at the inlet (Dirichlet condition) and, at the outlet, homogeneous Neumann conditions for the velocity components, are imposed. Pressure is fixed only at the outlet and is extrapolated at the other boundaries. On the vessel wall, no-slip Dirichlet conditions for the velocity field are prescribed. Since at time $t=0$ no clot exists, an undisturbed fully developed non-Newtonian flow is conveniently computed and imposed as initial condition. This undisturbed flow is also used as initial condition for the biochemistry simulations.

\subsubsection{Concentrations}

The vessel wall is impermeable and the boundary conditions for the concentrations of all the twenty-three chemical species, at the healthy vessel wall, are homogeneous of Neumann type (i.e. no-flux). In the injured wall region, no-flux boundary conditions are prescribed for all constituents except for seven selected chemical species which are directly involved in the initiation of the coagulation cascade [2-4]. Figure 9 represents this configuration.

The simulated vessel wall injury is considered to be a region which is the intersection of the outer surface of the vessel with a sphere of radius $R$, centered on the vessel wall

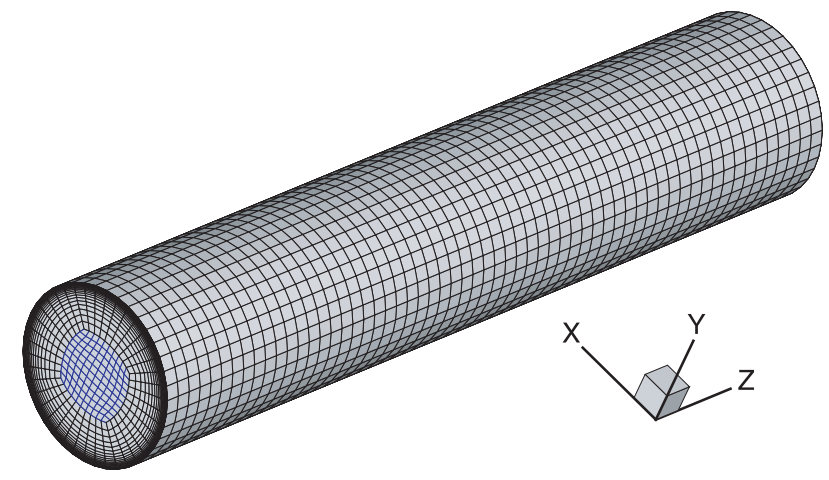

Figure 8. Computational geometry and grid. 
at one third of the length of the vessel segment. This means the clotting surface on the vessel wall is elliptical-shaped. In this region, non-homogeneous boundary conditions of the Neumann type $\partial \mathrm{C}_{i} / \partial \mathbf{n}=\mathrm{B}_{i}$ are prescribed for the following seven chemicals: IXa,IX, $X a, X, X I a, X I$ and $t P a$. These flux terms are summarized in Table 4 , where $[\mathrm{C}]^{\mathrm{w}}$ denotes the value of $C$ on the wall.

Diffusion coefficients $\mathrm{D}$ have already been listed in Table 2. The length scale $L$ is chosen to be equal to the vessel radius $R$. The other parameters appearing in the formulation of boundary fluxes are summarized in Table 5 .

Along with these constant type parameters, another three free parameters describing the level of the blood vessel wall injury appear and should be specified. These values are the $T F-V I I a$ complex concentration ([TF-VIIa]), the number of intact endothelial cells per unit area $[E N D O]$, and the XIIa enzyme concentration ([XIIa]). These values should be prescribed separately for injured and healthy vessel wall as shown in Figure 10 (using ??? and ??, respectively).

In addition, we require normal human physiological values as initial conditions for the concentrations of all chemical species (see Refs. [2-4]). These values are listed in Table 6. To avoid instabilities in the numerical simulations here, we assume that the initial concentrations of the enzymes originally set to zero (IXa, VIII $a, V a, X a, I I a$ and Ia) are replaced by suitable very small (but non-zero) values, set to be $0.1 \%$ of the initial values for the corresponding zymogens (IX, VIII, V, X, II and $I$ ). These free parameters are chosen to be equal to zero on the healthy part of the vessel wall, while on the injured area their prescribed values are shown in Table 7.

\subsection{Numerical results}

In this section, we present some of the results obtained with the three-dimensional numerical simulations of the coupled fluid-biochemistry model described above for the prediction of clot formation and growth around an injured vessel wall region, under physiological conditions. As already mentioned (Section 2.3), the initiation of the clotting cascade is associated to the extent of injury in the vessel wall, reflected in a threshold concentration of the TF-VIIa complex (tissue factor) imposed in the flux boundary conditions. The clot is formed when fibrin concentration $[I a]$ equals or exceeds a specific value $(1000 \mathrm{nM}$ in our case) and clot growth is determined by tracking in time the evolution of fibrin concentration in the clot region.

All the twenty-three chemical species involved in the advection-reaction-diffusion Equations (6) play an important role in the clotting process. Their concentrations are computed pointwise in the whole computational domain and can be visualized during

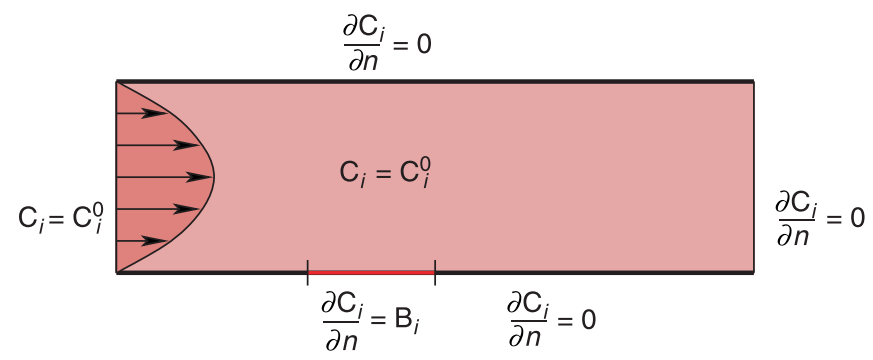

Figure 9. Concentration boundary conditions. 
Table 4. Flux boundary conditions at the injured vessel wall region.

\begin{tabular}{ll}
\hline Species & \multicolumn{1}{c}{ Expressions of the boundary flux terms $\mathrm{B}_{i}$} \\
\hline$I X a$ & $\left(\left(k_{7,9}[I X]^{w}[T F-V I I a]^{w}\right) /\left(K_{7,9 M}+[I X]^{w}\right)\right)\left(L / D_{I X a}\right)$ \\
$I X$ & $-\left(\left(k_{7,9}[I X]^{w}[T F-V I I a]^{w}\right) /\left(K_{7,9 M}+[I X]^{w}\right)\right)\left(L / D_{I X}\right)$ \\
$X a$ & $\left(\left(k_{7,10}[X]^{w}[T F-V I I a]^{w}\right) /\left(K_{7,10 M}+[X]^{w}\right)\right)\left(L / D_{X a}\right)$ \\
$X$ & $-\left(\left(k_{7,10}[X]^{w}[T F-V I I a]^{w}\right) /\left(K_{7,10 M}+[X]^{w}\right)\right)\left(L / D_{X}\right)$ \\
$X I a$ & $\left(\left(\phi_{11}[X I]^{w}[X I I a]^{w} /\left(\Phi_{11 M}+[X I]^{w}\right)\right)\left(L / D_{X I a}\right)\right.$ \\
$X I$ & $-\left(\left(\phi_{11}[X I]^{w}[X I I a]^{w}\right) /\left(\Phi_{11 M}+[X I]^{w}\right)\right)\left(L / D_{X I}\right)$ \\
$t P a$ & $\left(\left(k_{t P A}^{C}+k_{t P A}^{I I a}[I I a]^{w}+k_{t P A}^{I a}[I a]^{w}\right)[E N D O]^{w}\right)\left(L / D_{t P a}\right)$ \\
\hline
\end{tabular}

Table 5. Boundary flux parameters.

\begin{tabular}{lll}
\hline Parameters & \multicolumn{1}{c}{ Values } & \multicolumn{1}{c}{ Units } \\
\hline$\phi_{11}$ & 0.034 & $\mathrm{~min}^{-1}$ \\
$\Phi_{11 M}$ & 2000 & $\mathrm{nM}$ \\
$k_{7,9}$ & 32.4 & $\mathrm{nM}$ \\
$K_{7,9 M}$ & 24 & $\mathrm{nM}$ \\
$k_{7,10}$ & 103 & $\mathrm{~min}^{-1} \mathrm{~m}$ \\
$K_{7,10 M}$ & 240 & $\mathrm{nM}$ \\
$k_{t P A}^{C}$ & $6.52 \times 10^{-13}$ & $\mathrm{nM} \mathrm{m}^{2} \min ^{-1}$ \\
$k_{t P A}^{I I a}$ & $9.27 \times 10^{-12} \mathrm{e}^{-134.8\left(t-t_{0}\right)}$ & $\mathrm{m}^{2} \min ^{-1}$ \\
$k_{t P A}^{I a}$ & $5.059 \times 10^{-18}$ & $\mathrm{~m}^{2} \min ^{-1}$ \\
\hline
\end{tabular}

aselected simulation time. Figure 11 illustrates the evolution in time $(300 \mathrm{~s})$ of the concentrations of the six most important species involved in the blood coagulation, namely $[I I a]$ (Thrombin), [Ia] (Fibrin), [ATIII] (Anti-Thrombin III), [APC] (Active Protein C), $[t P A]$ (Tissue Plasminogen Activator) and [PLA] (Plasmin). The concentrations versus time history are shown at the centre of the simulated vessel wall injured region.

During the simulation time period of $300 \mathrm{~s}$ the concentrations of these chemicals undergo a profound evolution. The most relevant is the jump in all concentrations that occurs approximately $20 \mathrm{~s}$ after the initiation of the clotting cascade. At this time, the system response becomes stronger and the concentration of fibrin increases rapidly and reaches its maximum value at approximately $120 \mathrm{~s}$, remaining relatively stable after that time, Figure 11(b). Observing the different graphs shown in Figure 11, we also conclude that the time evolution is not similar for the various chemical species.

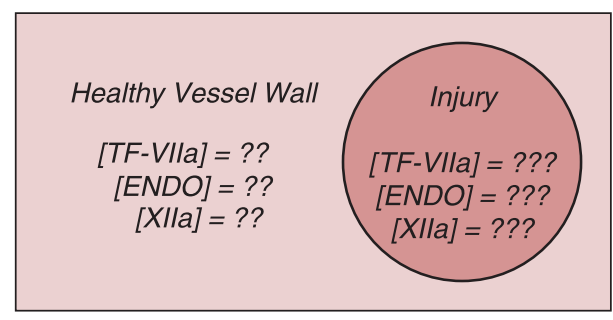

Figure 10. Free parameters in flux boundary conditions. 
Table 6. Initial concentrations.

\begin{tabular}{lc}
\hline Species & Initial values $(\mathrm{nM})$ \\
\hline$I X a$ & $0.0^{*}$ \\
$I X$ & $90.0^{*}$ \\
$V I I I a$ & $0.0^{*}$ \\
$V I I I$ & $0.7^{*}$ \\
$V a$ & $0.0^{*}$ \\
$V$ & $20.0^{*}$ \\
$X a$ & $0.0^{*}$ \\
$X$ & $170.0^{*}$ \\
$I I a$ & $0.0^{*}$ \\
$I I$ & 1400.0 \\
$I a$ & $0.0^{*}$ \\
$I$ & 7000.0 \\
$X I a$ & $0.0^{*}$ \\
$X I$ & 30.0 \\
$A T I I I$ & 2410.0 \\
$T F P I$ & 2.5 \\
$A P C$ & $0.0^{*}$ \\
$P C$ & 60.0 \\
$L 1 A T$ & 45000.0 \\
tPA & 0.08 \\
$P L A$ & $0.0^{*}$ \\
$P L S$ & 2180.0 \\
$L 2 A P$ & 105.0 \\
\hline &
\end{tabular}

Clot growth can be better observed in Figure 12, which shows the contours of fibrin concentration on the vessel wall after $60 \mathrm{~s}$. The simulations suggest that fibrin concentration has significantly increased in the clotting area. Moreover, due to advection, fibrin is transported downstream in the injured vessel wall region, as expected and the clot's shape changes its form during the clotting process.

These phenomena become more evident in Figure 13, where the variations of surface fibrin concentration contours during the initial $60 \mathrm{~s}$ of clotting are displayed. The length scales of both axes correspond to the axial and tangential coordinates, normalized by the vessel cross-section radius $R$. The blood flow direction is from left to right. In the first $20 \mathrm{~s}$, simulations do not show any change in the fibrin concentration and therefore only results for the time period 30-60 s are shown in Figure 13. Due to advection, fibrin is transported downstream in the injured vessel wall region, as already referred, and the clot's shape changes its form during the clotting process.

Advection effects are better captured with a longer simulation time of clot growth. This is shown in Figure 14 for the time period $0-300$ s. It is clear that the clot evolution process is not yet finished, as the clot shape is still changing. Also, clot dissolution that

Table 7. Free parameters in boundary fluxes.

\begin{tabular}{ll}
\hline Concentrations & \multicolumn{1}{c}{ Values } \\
\hline$[T F-V I I a]$ & $0.25 \mathrm{nM}$ \\
{$[E N D O]$} & $2.0 \times 10^{9}$ cells $/ \mathrm{m}^{2}$ \\
{$[X I I a]$} & $375 \mathrm{nM}$ \\
\hline
\end{tabular}



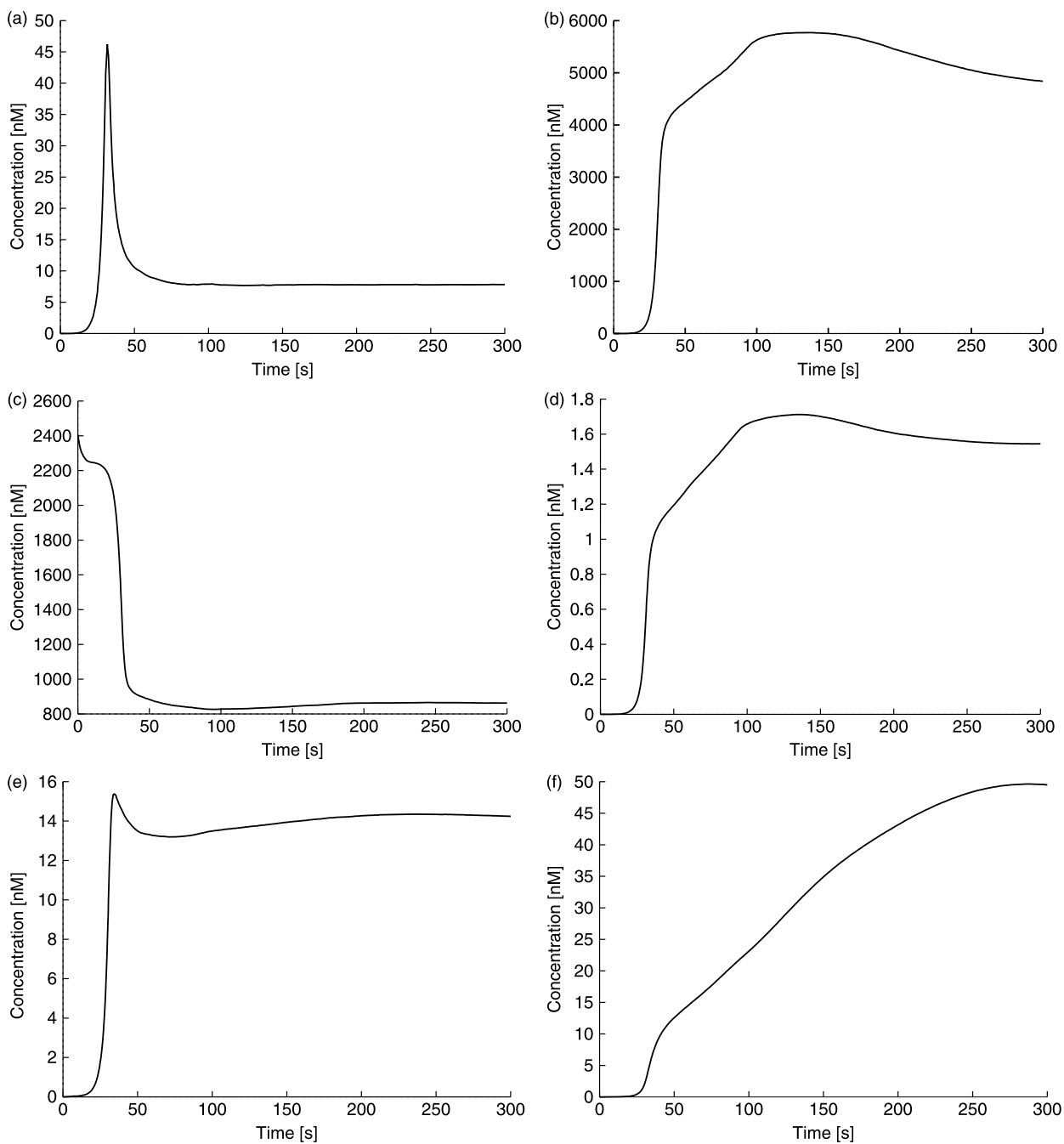

Figure 11. Time evolution of the concentrations of selected chemical species in the centre of the clotting surface. (a) Thrombin [IIa]; (b) Fibrin [Ia]; (c) Anti-Thrombin III [ATIII]; (d) Active Protein C [APC]; (e) Tissue Plasminogen [tPA]; (f) Plasmin [PLA].

occurs in regions where fibrin concentration drops below $\mathrm{C}_{\mathrm{Clot}}$, after initially exceeding it, or when the shear stress exceeds a critical value forcing the clot's rupture, has not been captured in the present simulations. A longer time clot growth and dissolution needs more computational time and is beyond the purpose of the preliminary numerical simulations performed in this study.

\section{Conclusion and remarks}

Preliminary numerical results of three-dimensional simulations for a simplified version of an advanced comprehensive model of clot growth and lysis, introduced and discussed in Refs. [2-4], have been presented here to emphasize its effectiveness. 


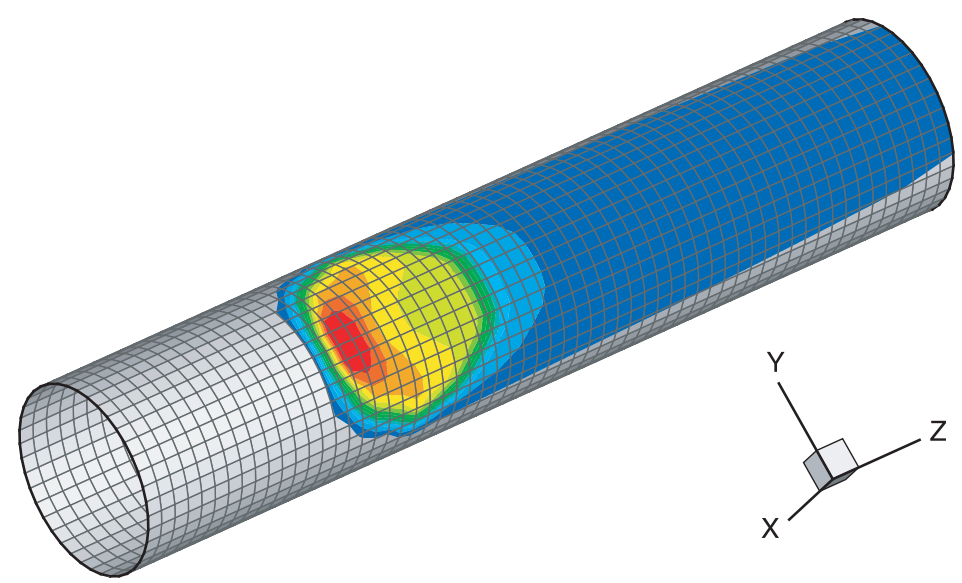

Figure 12. Fibrin concentration on the vessel surface after $60 \mathrm{~s}$.

The blood flow model used in the simulations only captures its shear-thinning viscosity and could be improved with more complex rheological models including viscoelasticity, as studied by Yeleswarapu [30], or the thermodynamically based model derived by Anand and Rajagopal [1] (with deformation dependent relaxation times) and used in Refs. [2-4]. Moreover, it would be interesting to incorporate in the solvers additional chemical constituents and their interactions, as in those involved in platelets activation and aggregation, to obtain numerical results for a more realistic coagulation model that fits physiological experimental data and may be used in clinical applications. Results can also
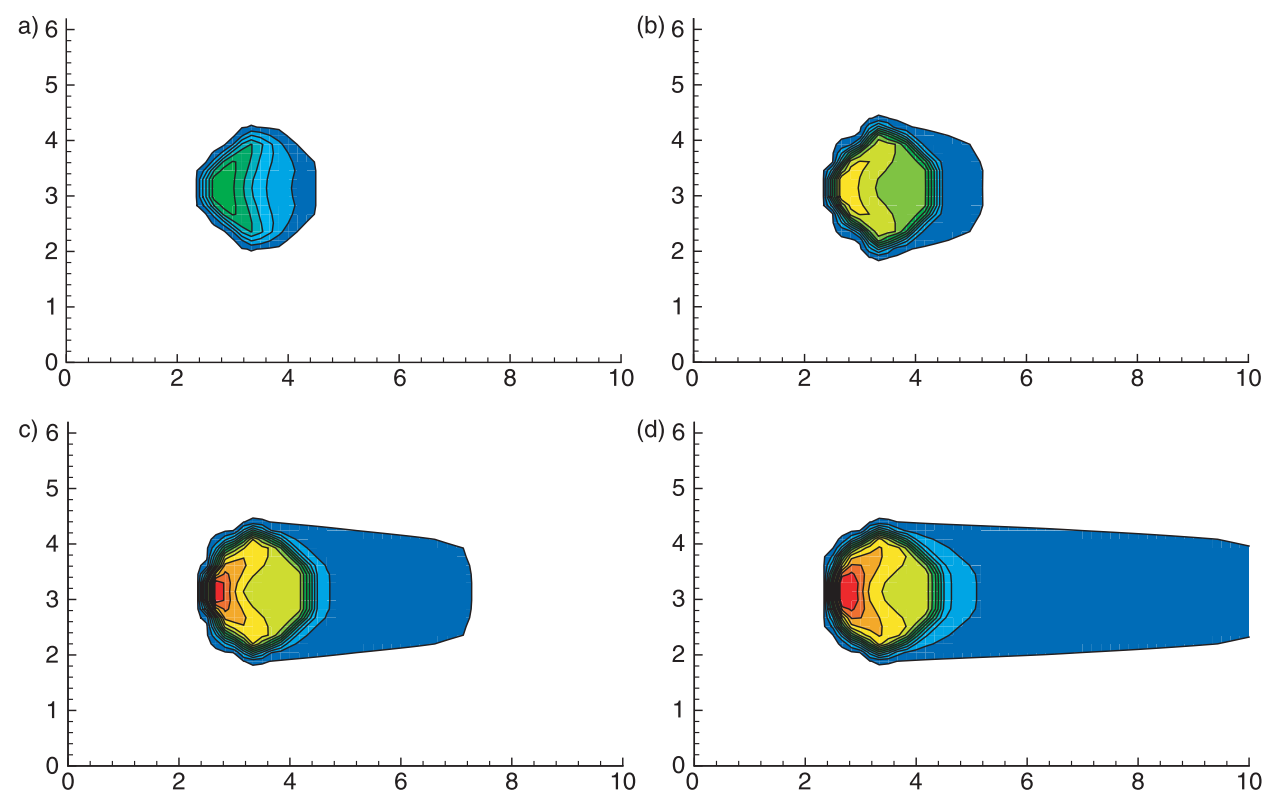

Figure 13. Evolution of fibrin concentrations on the vessel wall during the first minute of clotting. (a) $30 \mathrm{~s}$; (b) $40 \mathrm{~s}$; (c) $50 \mathrm{~s}$; (d) $60 \mathrm{~s}$. 

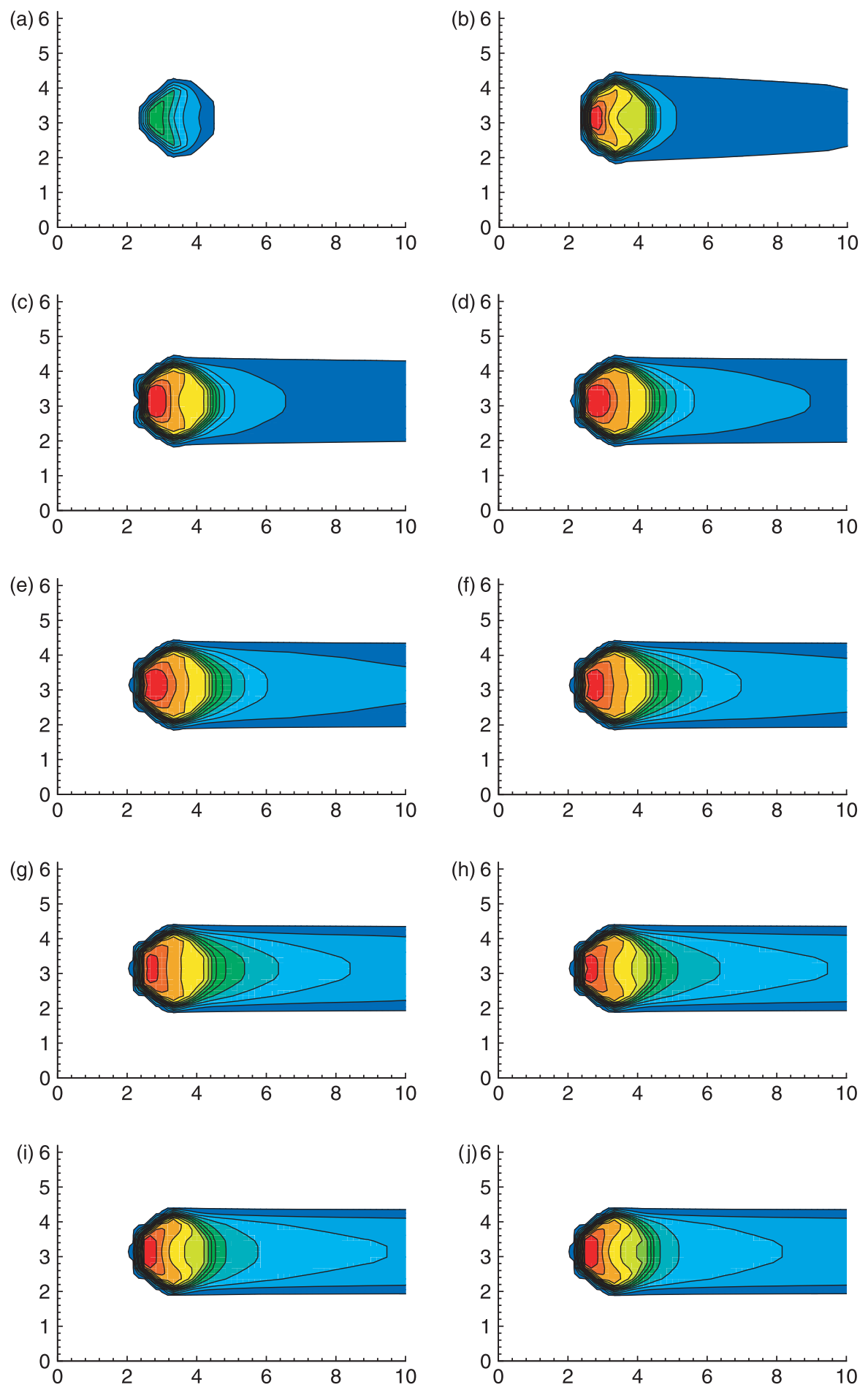

Figure 14. Evolution of fibrin concentrations on the vessel wall during the first 5 min of clotting. (a) $30 \mathrm{~s}$; (b) $60 \mathrm{~s}$; (c) $90 \mathrm{~s}$; (d) $120 \mathrm{~s}$; (e) $150 \mathrm{~s}$; (f) $180 \mathrm{~s}$; (g) $210 \mathrm{~s}$; (h) $240 \mathrm{~s}$; (i) $270 \mathrm{~s}$; (j) $300 \mathrm{~s}$. 
be improved using more efficient and robust space and time semi-discretization solvers that allow for the simulation of clot growth and dissolution with affordable computational costs. This is the object of our current research.

\section{Acknowledgements}

We are extremely thankful to Prof. K. R. Rajagopal for many stimulating discussions related to the mathematical model and its physiological meaning. This work has been partially supported by the European research project HPRN-CT-2002-002670, by the Research Plan MSM 6840770010 of the Ministry of Education of Czech Republic, by project PTDC/MAT/68166/2006 and by CEMAT-IST through FCT's funding program.

\section{References}

[1] M. Anand and K.R. Rajagopal, A shear-thinning viscoelastic fluid model for describing the flow of blood, Int. J. Cardiovas. Med. Sci. 4(2) (2004), pp. 59-68.

[2] M. Anand and K.R. Rajagopal, A model for the formation and lysis of clots in quiescent plasma. A comparison between the effects of antithrombin III deficiency and protein C deficiency, submitted (2007).

[3] M. Anand, K. Rajagopal, and K.R. Rajagopal, A model incorporating some of the mechanical and biochemical factors underlying clot formation and dissolution in flowing blood, J. Theor. Med. 5(3-4) (2003), pp. 183-218.

[4] M. Anand, K. Rajagopal, and K.R. Rajagopal, A model for the formation and lysis of blood clots, Pathophysiol. Haemost. Thromb. 34 (2005), pp. 109-120.

[5] F. Ataullakhanov, V. Zarnitsina, A. Pokhilko, A. Lobanov, and O. Morozova, Spatio-temporal dynamics of blood coagulation and pattern formation. A theoretical approach, Int. J. Bifur. Chaos 12(9) (2002), pp. 1985-2002.

[6] S. Butenas and K.G. Mann, Blood coagulation, Biochemistry (Moscow) 67(1) (2002), pp. 3-12 (translated from Biokhimiya, Vol. 67, No. 1, 2002, pp. 5-15.).

[7] S.E. Charm, and G.S. Kurland, Viscometry of human blood for shear rates of 0-100,000 $\mathrm{sec}^{-1}$, Nature 206 (1965), pp. 617-618.

[8] S. Chien, S. Usami, H.M. Taylor, J.L. Lundberg, and M.I. Gregersen, Effect of hematocrit and plasma proteins on human blood rheology at low shear rates, J. Appl. Physiol. 21 (1966), pp. 81-87.

[9] S. Chien, S. Usami, R. Dellenback, and M. Gregersen, Shear-dependent deformation of erythrocytes in rheology of human blood, Am. J. Physiol. 219 (1970), pp. 136-142.

[10] G. Colantuoni, J. Hellums, J. Moake, and C. Alfre, The response of human platelets to shear stress at short exposure times, Trans. Amer. Soc. Artificial Int. Organs 23 (1977), pp. 626-631.

[11] B. Engquist, P. Lötstedt, and B. Sjögreen, Nonlinear filters for efficient shock computation, Math. Comput. 52(186) (1989), pp. 509-537.

[12] A.L. Fogelson, Continuum models of platelet aggregation: Formulation and mechanical properties, SIAM J. Appl. Math. 52 (1992), pp. 1089-1110.

[13] A. Jameson, Time dependent calculations using multigrid, with applications to unsteady flows past airfoils and wings, in Proceedings of the AIAA 10th Computational Fluid Dynamics Conference, Honolulu. 1991, June (aIAA Paper 91-1596).

[14] A. Jameson, W. Schmidt, and E. Turkel, Numerical solutions of the Euler equations by finite volume methods using Runge-Kutta time-stepping Schemes, in Proceedings of the AIAA 14th Fluid and Plasma Dynamic Conference, Palo Alto, CA. 1981, June (aIAA paper 81-1259).

[15] H. Kroll, J. Hellums, L. McIntire, A.I. Schafer, and J. Moake, Platelets and shear stresss, Blood 88 (1996), pp. $1525-1541$.

[16] A.L. Kuharsky and A.L. Fogelson, Surface-mediated control of blood coagulation: The role of binding site densities and platelet deposition, Biophys. J. 80 (2001), pp. 1050-1074.

[17] A. Leuprecht and K. Perktold, Computer simulation of non-Newtonian effects of blood flow in large arteries, Comput. Meth. Biomech. Biomech. Eng. 4 (2001), pp. 149-163.

[18] K. Mann, K. Brummel-Ziedins, T. Orfeo, and S. Butenas, Models of blood coagulation, Blood Cells, Mol. Dis. 36 (2006), pp. 108-117.

[19] S. Nägele and G. Wittum, On the influence of different stabilisation methods for the incompressible NavierStokes equations, J. Comp. Phys. 224 (2007), pp. 100-116.

[20] K. Rajagopal and J. Lawson, Regulation of hemostatic system function by biochemical and mechanical factors, in Modeling of Biological Materials. Birkhäuser, Boston, 2007, pp. 179-210.

[21] P. Riha, X. Wang, R. Liao, and J. Stoltz, Elasticity and fracture strain of blood clots, Clin. Hemorheol. Microcirc. 21(1) (1999), pp. 45-49. 
[22] A. Robertson, A. Sequeira, and M. Kameneva, Hemorheology, in Hemodynamical Flows: Modelling, Analysis and Simulation. Oberwolfach Seminars Series. Birkhäuser, Boston, 2008.

[23] A. Robertson, A. Sequeira, and R. Owens, Rheological models for blood, in Cardiovascular Mathematics. Springer, Berlin (to appear) 2008.

[24] M. Schenone and B. Furie The blood coagulation cascade, Curr. Opin. Hematol. 11 (2004), pp. 272-277.

[25] H. Schmid-Schönbein and R. Wells, Fluid drop-like transition of erythrocytes under shear, Science 165 (1969), pp. 288-291.

[26] W. Shyy, M.H. Chen, R. Mittal, and H.S. Udaykumar, On the suppression of numerical oscillations using a non-linear filter, J. Comput. Phys. 102 (1992), pp. 49-62.

[27] G.B. Thurston, Frequency and shear rate dependence of viscoelasticity of human blood, Biorheology 10 (1973), pp. 375-381.

[28] J. Vierendeels, K. Riemslagh, and E. Dick, A multigrid semi-implicit line-method for viscous incompressible and low-Mach-number flows on high aspect ratio grids, J. Comput. Phys. 154 (1999), pp. 310-344.

[29] N. Wang and A.L. Fogelson, Computational methods for continuum models of platelet aggregation, J. Comput. Phys. 151 (1999), pp. 649-675.

[30] K. Yeleswarapu, M. Kameneva, K. Rajagopal, and J. Antaki, The flow of blood in tubes: Theory and experiments, Mech. Res. Commun. 25(3) (1998), pp. 257-262.

[31] V.I. Zarnitsina, A.V. Pokhilko, and F.I. Ataullakhanov, A mathematical model for the spatio-temporal dynamics of intrinsic pathway of blood coagulation - I. The model description, Thromb. Res. 84(4) (1996a), pp. $225-236$.

[32] V.I. Zarnitsina, A.V. Pokhilko, and F.I. Ataullakhanov, A mathematical model for the spatio-temporal dynamics of intrinsic pathway of blood coagulation - II. Results, Thromb. Res. 84(5) (1996b), pp. 333-344. 


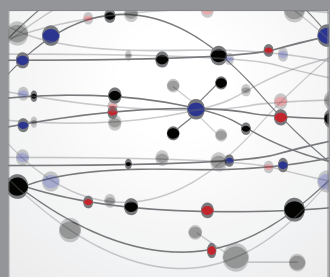

The Scientific World Journal
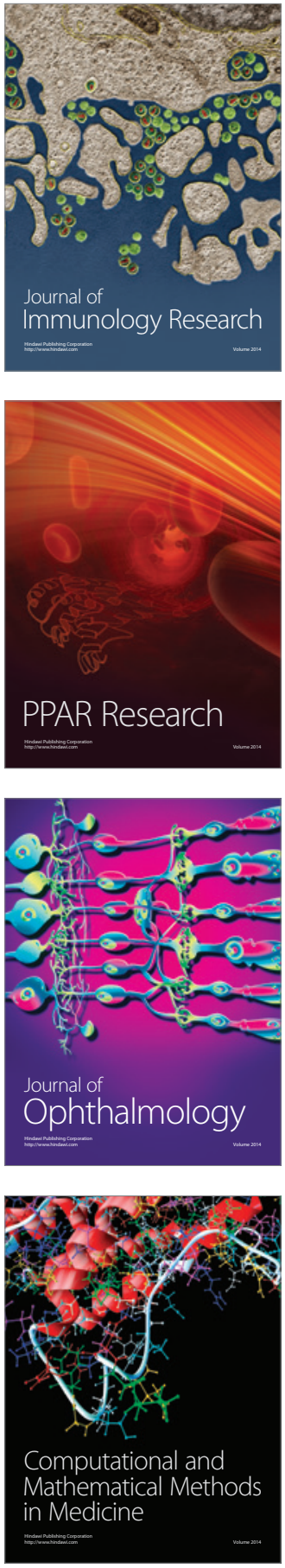

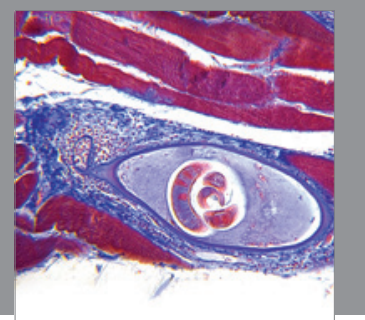

Gastroenterology

Research and Practice
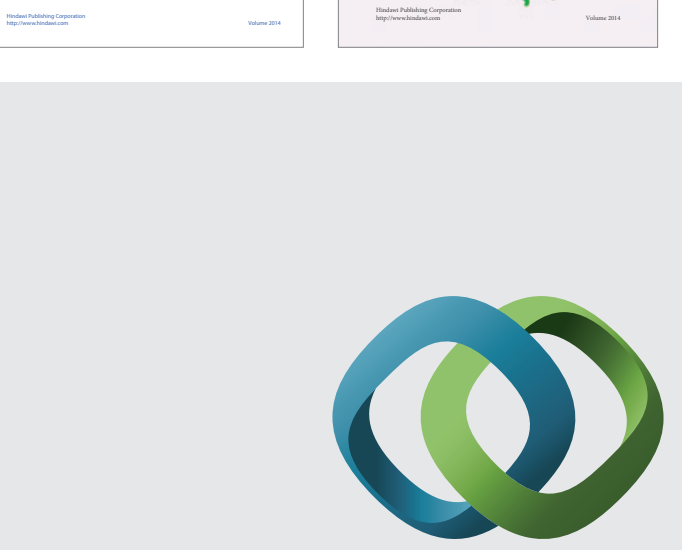

\section{Hindawi}

Submit your manuscripts at

http://www.hindawi.com
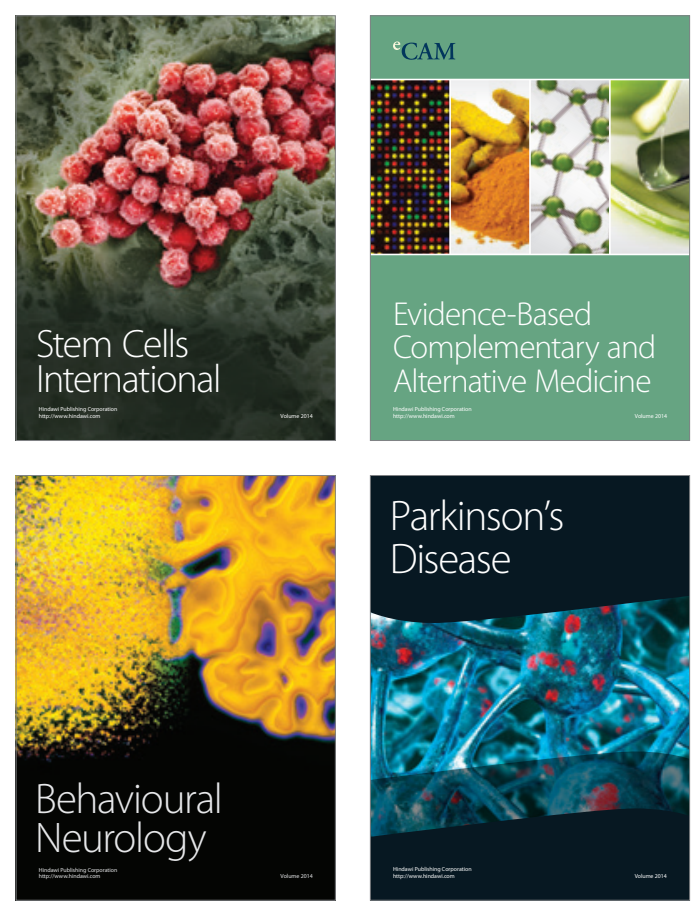

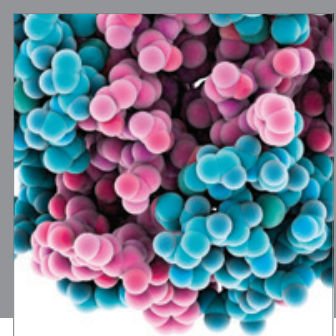

Journal of
Diabetes Research

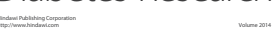

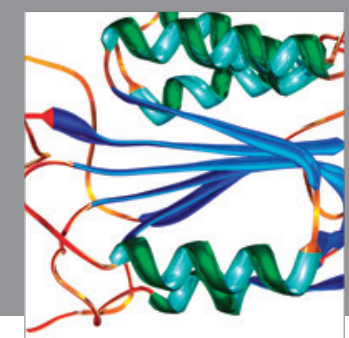

Disease Markers
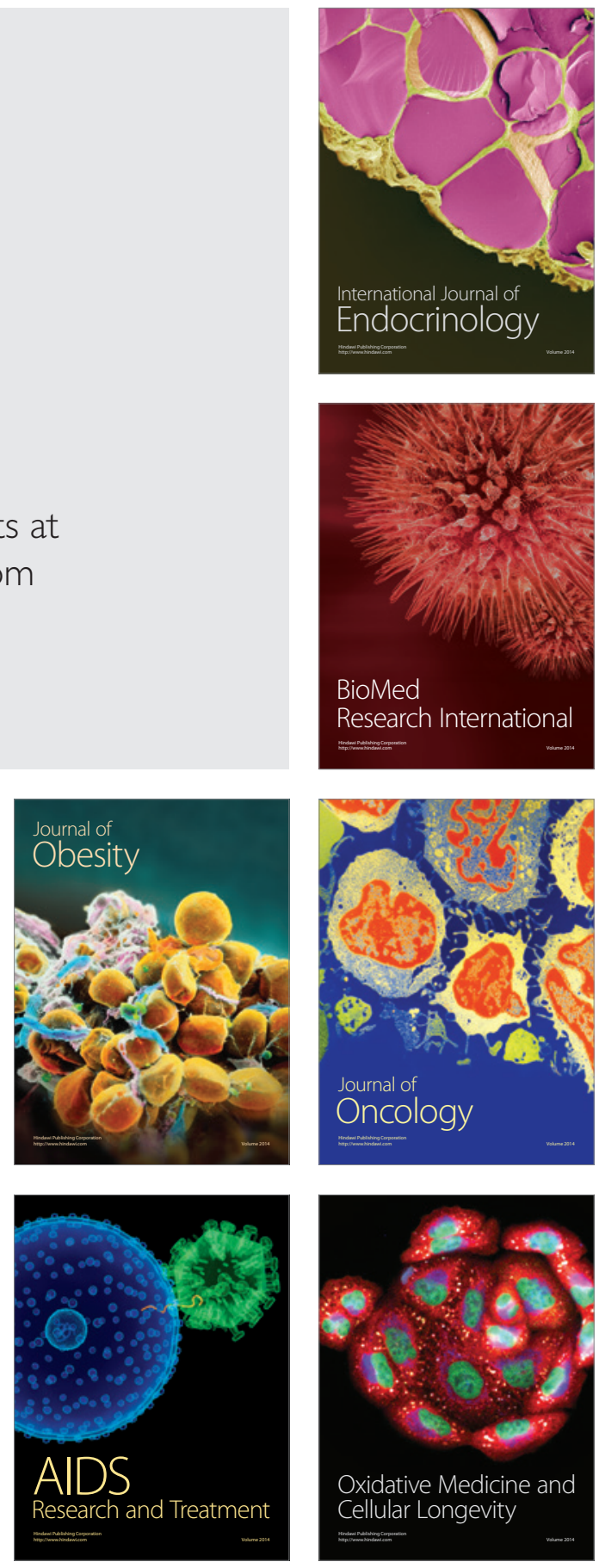